\title{
On effective action of multiple M5-branes and ABJM action
}

\author{
Seiji Terashima ${ }^{a}$ and Futoshi Yagi ${ }^{b}$ \\ ${ }^{a}$ Yukawa Institute for Theoretical Physics, Kyoto University, \\ Kyoto 606-8502, Japan \\ ${ }^{b}$ Institut des Hautes Études Scientifiques, \\ Bures-sur-Yvette, 91440, France \\ E-mail: terasima@yukawa.kyoto-u.ac.jp, fyagi@ihes.fr
}

ABSTRACT: We calculate the fluctuations from the classical multiple M5-brane solution of ABJM action which we found in the previous paper. We obtain D4-brane-like action but the gauge coupling constant depends on the spacetime coordinate. This is consistent with the expected properties of M5-brane action, although we will need to take into account the monopole operators in order to fully understand M5-branes. We also see that the Nambu-Poisson bracket is hidden in the solution.

Keywords: Brane Dynamics in Gauge Theories, p-branes, M-Theory

ArXIV EPRINT: 1012.3961 


\section{Contents}

1 Introduction 1

2 ABJM action and the M5-brane solution 2

2.1 ABJM action 2

2.2 M5-brane solution 4

3 The M5-action from the ABJM action $\quad 6$

$\begin{array}{ll}3.1 & \text { Expansion of the bosonic potential }\end{array}$

3.2 Expansion of the ABJM action 8

$\begin{array}{ll}3.3 & \text { Action of the fluctuations around the solution } \\ \end{array}$

$\begin{array}{lll}4 & \text { Discussion } & 13\end{array}$

\section{Introduction}

Understanding the dynamics of M5-branes is one of the most important problems in Mtheory or string theory. For the D-branes, the low energy effective actions were found and they are essentially the Yang-Mills action. However, the effective action for the multiple M5-branes is not known yet, although single M5-brane action is known [1-3]. Actually, from the AdS/CFT correspondence, the degree of freedom of the $N$ M5-branes will be proportional to $N^{3}$, which can not be realized by the Yang-Mills theory naively. Thus, there should be interesting physics which is not yet known behind it.

Recently, the effective action of multiple M2-branes on $\mathbb{C}^{4} / \mathbb{Z}_{k}$ was suggested by [4] (we will call the action as ABJM action), following [5-8]. Because the D4-brane action is constructed from the D2-brane action by the Matrix theory like construction $[9,10]$ using the non-commutative space [11]-[15], we expect that this ABJM action will be useful to study M5-branes. More concretely, it is known that the effective action of $N$ D2-branes, which is three dimensional supersymmetric Yang-Mills theory, in a large $N$ limit has a classical solution which correspond to D4-branes with a constant magnetic flux. This is an D2-D4 bound state and the flux represents the non-zero D2-brane charge. When we consider small fluctuation around this classical solution, we obtain the effective D4-brane action, which is five dimensional supersymmetric Yang-Mills theory. We would like to uplift this situation to M-theory in order to obtain an M5-brane action from an M2-brane action, which we expect to be the ABJM action. ${ }^{1}$

Several classical solutions of M2-M5 bound state have been found in the ABJM action. One of them is the M2-branes ending on M5-branes. Such a classical solution was first

\footnotetext{
${ }^{1}$ In $[16,17]$, a single M5-brane is constructed from BLG model by using Nambu-Poisson bracket as the three-algebra. However, multiple M5-branes has not been obtained.
} 
studied in [18] and a closely related solution was also found in the mass deformed ABJM action [19]. ${ }^{2}$ Although both of them are expected to form a fuzzy $S^{3} / \mathbb{Z}_{k}$, they actually show a fuzzy $S^{2}$ in the naive classical analysis. The fluctuation around this classical solution was also calculated [21] and shown that it actually reduces to D4-brane action. ${ }^{3}$ Indeed, the classical solution [19] of the mass deformed ABJM model is shown to be exactly equivalent to the usual fuzzy $S^{2}$ solution corresponding to the D2-D4 bound state constructed with the adjoint scalar field at the classical level ${ }^{4}[21]$.

In the previous paper [22], we found another classical solution of M5-branes. This solution is an uplift of the flat D4-brane solution with a constant magnetic flux, which is constructed from infinitely many D2-branes ${ }^{5}$ satisfying $\left[X^{1}, X^{2}\right]=$ const. where $X^{1}$ and $X^{2}$ are the adjoint scalar fields corresponding to the transverse direction of the D2-branes like [9]. The three-algebra structure was also found in this solution. In this paper, we expand ABJM action around our classical solution. We obtain D4-brane like action, which contains only the zero modes of the $S^{1}$ direction on which the $\mathbb{Z}_{k}$ of the $\mathbb{C}^{4} / \mathbb{Z}_{k}$ acts. This is because the non-zero modes should have the vortex (or monopole) charge through the Chern-Simons term. In order to include them, we should take into account the monopole operators [4].

However, it would be remarkable that the gauge coupling constant of our D4-brane like action depends on the spacetime coordinate. We would like to stress that such an action is not obtained from the D2-branes. This dependence reflects the geometry $\mathbb{C}^{4} / \mathbb{Z}_{k}$ in which the radius of the $S^{1}$ increases as we go away from the orbifold fixed point. In this sense, our action includes the information of M5-brane, which is not included in the D4-brane constructed from D2-branes. Although our result may not include all the low energy dynamics of the M5-branes, we hope it will still be helpful for the understanding of the M5-branes.

\section{ABJM action and the M5-brane solution}

In this section, we review ABJM action and our classical M5-brane solution. Then, we discuss that the structure of Nambu-Poisson bracket is hidden in our classical solution.

\subsection{ABJM action}

ABJM action is a three dimensional $N=6$ supersymmetric Chern-Simons theory, whose gauge group is $\mathrm{U}(N)_{1} \times \mathrm{U}(N)_{2}$. Its matter contents are gauge fields $A_{\mu}^{(1)}, A_{\mu}^{(2)}$ of each gauge group, four complex bi-fundamental scalar fields $Y^{A}(A=1,2,3,4)$ and their fermionic

\footnotetext{
${ }^{2}$ For still another closely related work, see also [20].

${ }^{3}$ It is discussed in [21] that we have to take the limit $k \rightarrow \infty$ in order that this analysis is reliable. In this limit, M5-branes reduce to D4-branes.

${ }^{4}$ Since this analysis is purely classical, the contribution from the monopole operator, which we mention later, is not included.

${ }^{5}$ Since this D4-brane solution exists in the strict large $N$ limit contrary to the case of the fuzzy sphere which has a finite volume, so does our M5-brane solution. In this paper, our discussion is limited to the case where the number of the M2-branes is strictly infinite.
} 
superpartners. The bosonic part of the ABJM action is given by

$$
\begin{array}{r}
L=\frac{k}{4 \pi} \varepsilon^{\mu \nu \rho} \operatorname{Tr}\left(A_{\mu}^{(1)} \partial_{\nu} A_{\lambda}^{(1)}+\frac{2 i}{3} A_{\mu}^{(1)} A_{\nu}^{(1)} A_{\lambda}^{(1)}-A_{\mu}^{(2)} \partial_{\nu} A_{\lambda}^{(2)}-\frac{2 i}{3} A_{\mu}^{(2)} A_{\nu}^{(2)} A_{\lambda}^{(2)}\right) \\
-\operatorname{Tr}\left[\left(\tilde{D}_{\mu} Y_{A}\right)^{\dagger} \tilde{D}^{\mu} Y^{A}\right]-V_{\mathrm{bos}}
\end{array}
$$

The bosonic potential $V_{\mathrm{bos}}$ is given by

$$
V_{\mathrm{bos}}=-\frac{4 \pi^{2}}{3 k^{2}} \operatorname{Tr}\left(\left\{\Upsilon^{A B}{ }_{C}, \Upsilon_{A B}^{C}\right\}\right)
$$

where

$$
\Upsilon^{A B}{ }_{C}=\left[Y^{A}, Y^{B} ; Y_{C}\right]-\frac{1}{2} \delta_{C}^{A}\left[Y^{D}, Y^{B} ; Y_{D}\right]+\frac{1}{2} \delta_{C}^{B}\left[Y^{D}, Y^{A} ; Y_{D}\right]
$$

and the bracket $\{$,$\} is the anti-commutator. Here, the three-bracket is defined as$

$$
[X, Y ; Z]=X Z Y-Y Z X .
$$

The scalar fields with lower indices are given by

$$
Y_{A}=\left(Y^{A}\right)^{\dagger}
$$

By using the definition above, the bosonic potential can be explicitly written in terms of three-bracket as

$$
\begin{aligned}
V_{\mathrm{bos}}=-\frac{4 \pi^{2}}{3 k^{2}} \operatorname{Tr}(\{ & {\left.\left[Y^{A}, Y^{B} ; Y_{C}\right],\left[Y_{A}, Y_{B} ; Y^{C}\right]\right\} } \\
& \left.-\frac{1}{2}\left\{\left[Y^{A}, Y^{C} ; Y_{A}\right],\left[Y_{B}, Y_{C} ; Y^{B}\right]\right\}\right) .
\end{aligned}
$$

For later convenience, we define new basis of the gauge fields as

$$
A_{\mu} \equiv \frac{1}{2}\left(A_{\mu}^{(1)}+A_{\mu}^{(2)}\right), \quad B_{\mu} \equiv \frac{1}{2}\left(A_{\mu}^{(1)}-A_{\mu}^{(2)}\right) .
$$

Rewriting the Chern-Simons term with these new basis, we obtain

$$
L_{\mathrm{CS}}=\frac{k}{2 \pi} \varepsilon^{\mu \nu \rho} \operatorname{Tr}\left(B_{\mu} F_{\nu \lambda}+\frac{2 i}{3} B_{\mu} B_{\nu} B_{\lambda}\right),
$$

where we put

$$
F_{\nu \lambda}=\partial_{\nu} A_{\lambda}-\partial_{\nu} A_{\lambda}+i\left[A_{\nu}, A_{\lambda}\right] .
$$

The covariant derivatives for the bi-fundamental fields $Y^{A}$ are also rewritten in terms (2.7) as

$$
\tilde{D}_{\mu} Y^{A}=D_{\mu} Y^{A}+i\left\{B_{\mu}, Y^{A}\right\}
$$

where we put

$$
D_{\mu} Y^{A}=\partial_{\mu} Y^{A}+i\left[A_{\mu}, Y^{A}\right]
$$


The bosonic part of the ABJM action is then rewritten as

$$
\begin{aligned}
L= & \frac{k}{2 \pi} \varepsilon^{\mu \nu \rho} \operatorname{Tr}\left(B_{\mu} F_{\nu \lambda}+\frac{2 i}{3} B_{\mu} B_{\nu} B_{\lambda}\right) \\
& -\operatorname{Tr}\left(D_{\mu} Y_{A}+i\left\{B_{\mu}, Y_{A}\right\}\right)^{\dagger}\left(D^{\mu} Y^{A}+i\left\{B^{\mu}, Y^{A}\right\}\right)-V_{\mathrm{bos} .}
\end{aligned}
$$

The moduli space of this theory is $\left(\mathbb{C}^{4} / \mathbb{Z}_{k}\right)^{N} / S_{N}$, where $\mathbb{Z}_{k}$ simultaneously rotate the phase of all the complex scalar fields $Y^{i}$ by $2 \pi / k$. Thus, the ABJM model is supposed to describe the $N$ M2-branes probing $\mathbb{C}^{4} / \mathbb{Z}_{k}$. If we take the limit $k \rightarrow \infty$ and look far away from the orbifold fixed point at the same time, the local geometry of $\mathbb{C}^{4} / \mathbb{Z}_{k}$ becomes cylinder. Thus, the M2-branes can be regarded as D2-branes probing $\mathbb{R}^{7}$ in this limit. Indeed, when we give a vacuum expectation value $v$ to one of the scalars $Y^{i}$ and expand around that vacuum, and consider the following limit;

$$
k, v \rightarrow \infty \text { with } \quad \frac{k^{2}}{32 \pi^{2} v^{2}}=\frac{1}{4 g_{Y M}^{2}} \text { fixed }
$$

we obtain the D2-branes low energy effective action, i.e. the super Yang-Mills theory [4, 23]. Due to the Higgs mechanism, the field $B_{\mu}$ becomes massive and integrated out while $A_{\mu}$ remains as a gauge field on the D2-branes. We denote the limit (2.13) as the scaling limit in this paper.

\subsection{M5-brane solution}

In the previous paper [22], we showed the existence and uniqueness, up to some trivial ambiguities, of the solution of the following form of the equations of motion for $\mathrm{U}(N) \times \mathrm{U}(N)$ ABJM action with $N \rightarrow \infty$ :

$$
\begin{aligned}
& Y^{1}=Y_{1}=1_{n \times n} \otimes r(\hat{x}, \hat{y}), \quad Y^{2}=Y_{2}=1_{n \times n} \otimes r^{\prime}(\hat{x}, \hat{y}), \\
& Y^{3}=0, \quad Y^{4}=0, \\
& A_{\mu}^{(1)}=A_{\mu}^{(2)}=0,
\end{aligned}
$$

where

$$
r(\hat{x}, \hat{y})=v+\hat{x}+\mathcal{O}\left(v^{-1}\right), \quad r^{\prime}(\hat{x}, \hat{y})=\hat{y}, \quad[\hat{x}, \hat{y}]=i \Theta,
$$

and we regard that $\hat{x}$ and $\hat{y}$ are infinite dimensional irreducible hermitian matrices. This solution is constructed so that it reduces in the scaling limit to the solution representing the $n$ D4-branes in the action of infinitely many D2-branes. Thus, we interpret this classical solution as a solution representing $n$ M5-branes. We found the explicit form of $r(\hat{x}, \hat{y})$ pertubatively in $\mathcal{O}\left(v^{-9}\right)[22]$.

Interestingly, in the commutative limit $\Theta \rightarrow 0,{ }^{6}$ we can replace the commutator by Poisson bracket and found the solution:

$$
\left[r, r^{\prime}\right]_{P}=\frac{i \Theta v}{\sqrt{r^{2}+r^{\prime 2}}}
$$

\footnotetext{
${ }^{6}$ This limit will correspond to the large background magnetic field in the D4-brane picture. The reason why the vanishing magnetic field limit does not correspond to $\Theta \rightarrow 0$ limit is that we use matrix model like construction of the solution [15].
} 
where $[,]_{P}$ represents the Poisson bracket and $v$ is a constant. ${ }^{7}$ Here the coordinates become commutative in the limit and we denote them as $r$, and $r^{\prime}$. More explicitly, from the above equation with the ansatz, the function $r(x, y)$ is determined by

$$
\frac{\partial r(x, y)}{\partial x}=\frac{v}{\sqrt{r^{2}+y^{2}}}
$$

with

$$
[x, y]_{P}=i \Theta .
$$

Note that the M5-branes span $\left\{r, r^{\prime}, \theta\right\}$ where $Y^{1}=r e^{i \theta}$ and $Y^{2}=r^{\prime} e^{i \theta}$. The $\theta$-direction corresponds to the $S^{1}$ direction which is mentioned in section 1. The induced metric on the M5-branes is given by

$$
d s^{2}=d s_{(3)}^{2}+d r^{2}+d r^{2}+\left(r^{2}+r^{2}\right) d \theta^{2},
$$

where $d s_{(3)}^{2}$ is the flat metric of the $1+2$ dimensional Minkowski space-time and then, the Nambu-Poisson bracket naturally defined as $[x, y, \theta]_{N P} \sim \frac{1}{\sqrt{\operatorname{det} g_{i j}}} \epsilon^{i j k} \partial_{i} x \partial_{j} y \partial_{k} \theta$ where $i, j, k=\{r, y, \theta\}$ on this space is constant. However, if we naively define a Poisson bracket on the dimensionally reduced space with $d s^{2}=d r^{2}+d r^{\prime 2}$ as $[x, y]_{P}^{\prime} \sim \frac{1}{\sqrt{\operatorname{det} g_{i j}}} \epsilon^{i j} \partial_{i} x \partial_{j} y$ where $i, j=\{r, y\}$, then it is not constant by (2.18) and is different from (2.19).

Thus, the above Poisson bracket will have to be regarded as the Nambu-Poisson bracket with one variable always chosen to $\theta$,

$$
\left[f\left(r, r^{\prime}\right), g\left(r, r^{\prime}\right)\right]_{P}=\left[f\left(r, r^{\prime}\right), g\left(r, r^{\prime}\right), \theta\right]_{N P},
$$

where

$$
\left[r, r^{\prime}, \theta\right]_{N P}=\frac{i \Theta v}{\sqrt{r^{2}+r^{\prime 2}}}
$$

with

$$
[x, y, \theta]_{N P}=i \Theta
$$

It can be written as

$$
\left[r, r^{\prime}, \theta\right]_{N P}=i \Theta v{\sqrt{\operatorname{det} g_{i j}}}^{-1},
$$

where $g_{i j}$ is the metric on the space spanned by $\left\{r, r^{\prime}, \theta\right\}$.

In the following section, we consider fluctuations from this classical solution ${ }^{8}$ in the commutative limit $\Theta \rightarrow 0$.

\footnotetext{
${ }^{7}$ The anti-bracket $\{f, g\}$ will be approximated as $2 f g$ using the star-product formalism in the limit and the equations of motion become

$$
\left.\left.0 \simeq\left(\left(Y^{1}\right)^{2}\right)+\left(Y^{2}\right)^{2}\right)\right)\left[Y^{2},\left[Y^{1}, Y^{2}\right]_{P}\right]_{P}-Y^{1}\left(\left[Y^{1}, Y^{2}\right]_{P}\right)^{2}
$$$$
\left.\left.0 \simeq\left(\left(Y^{1}\right)^{2}\right)+\left(Y^{2}\right)^{2}\right)\right)\left[Y^{1},\left[Y^{1}, Y^{2}\right]_{P}\right]_{P}+Y^{2}\left(\left[Y^{1}, Y^{2}\right]_{P}\right)^{2} .
$$

The general solution of these equations is indeed (2.16).

${ }^{8}$ It is more appropriate to express the solution as $\left[r, r^{\prime}, \theta\right]_{N P}=\frac{C}{\sqrt{r^{2}+r^{\prime 2}}}$, where $C$ is a constant which represents a strength of the background 3-form field strength. If we focus on the solution near $r=v$, then the non-commutative parameter $\Theta$ of the effective D5-brane action is $v$-dependent as $\Theta=C / v$.
} 


\section{The M5-action from the ABJM action}

In this section, we will expand the ABJM action around our classical solution (2.16) and find the action for the multiple M5-branes, although we can keep only the zero-mode for the $\theta$ direction in the action. We will see the action has a form of the Yang-Mills action with a space-time dependent gauge coupling constant.

\subsection{Expansion of the bosonic potential}

Before expanding the ABJM action around our classical solution, we rewrite the bosonic potential (2.6) for later convenience. Since we will later use the classical solution (2.16), which is valid in the commutative limit, we expand the potential term by the number of the commutators. As will be explained later, we will take the fluctuations such that one commutator in the potential is $\mathcal{O}(\Theta)$.

First, we rewrite the three-bracket by using commutator and anti-commutator as

$$
\left[Y^{A}, Y^{B} ; Y_{C}\right]=\frac{1}{2}\left(\left\{\left[Y^{A}, Y^{B}\right], Y_{C}\right\}+\left\{\left[Y^{A}, Y_{C}\right], Y^{B}\right\}-\left\{\left[Y^{B}, Y_{C}\right], Y^{A}\right\}\right),
$$

which is shown by using the graded Jacobi identity

$$
[\{A, B\}, C]+\{[C, A], B\}-\{[B, C], A\}=0 .
$$

Because the three-bracket can be represented as (3.1), the leading terms are terms with two commutators. By substituting (3.1) into (2.6), we find that the potential is given by

$$
\begin{aligned}
V_{\mathrm{bos}}= & -\frac{4 \pi^{2}}{3 k^{2}} \operatorname{Tr}\left(\left\{\left[Y^{A}, Y^{B} ; Y_{C}\right],\left[Y_{A}, Y_{B} ; Y^{C}\right]\right\}-\frac{1}{2}\left\{\left[Y^{A}, Y^{C} ; Y_{A}\right],\left[Y_{B}, Y_{C} ; Y^{B}\right]\right\}\right) \\
= & -\frac{4 \pi^{2}}{3 k^{2}} \operatorname{Tr}\left(|Y|^{2}\left(-2\left|\left[Y^{A}, Y^{B}\right]\right|^{2}-4\left|\left[Y^{A}, Y_{C}\right]\right|^{2}-\left|\left[Y^{A}, Y_{A}\right]\right|^{2}\right)\right. \\
& +5\left|\left[Y^{B}, Y_{A}\right] Y^{A}\right|^{2}+\left|\left[Y^{B}, Y^{A}\right] Y_{A}\right| \\
& \left.+3\left(\left[Y^{A}, Y^{B}\right] Y_{B}\left[Y_{A}, Y^{C}\right] Y_{C}+\left[Y_{A}, Y_{B}\right] Y^{B}\left[Y^{A}, Y_{C}\right] Y^{C}\right)-2 Y_{c}\left[Y^{C}, Y_{A}\right] Y^{A}\left[Y^{B}, Y_{B}\right]\right) \\
& +\mathcal{O}\left([,]^{3}\right) \\
= & -\frac{4 \pi^{2}}{3 k^{2}} \operatorname{Tr}(3 A+B+3 C+2 D)+\mathcal{O}\left([,]^{3}\right),
\end{aligned}
$$

where we have defined

$$
\begin{aligned}
& A=-|Y|^{2}\left(\left|\left[Y^{A}, Y^{B}\right]\right|^{2}+\left|\left[Y^{A}, Y_{C}\right]\right|^{2}\right), \\
& B=|Y|^{2}\left(\left|\left[Y^{A}, Y^{B}\right]\right|^{2}-\left|\left[Y^{A}, Y_{B}\right]\right|^{2}-\left|\left[Y^{A}, Y_{A}\right]\right|^{2}\right), \\
& C=\left|\left[Y^{B}, Y_{A}\right] Y^{A}\right|^{2}+\left|\left[Y^{B}, Y^{A}\right] Y_{A}\right|^{2}+\left[Y^{A}, Y^{B}\right] Y_{B}\left[Y_{A}, Y^{C}\right] Y_{C}+\left[Y_{A}, Y_{B}\right] Y^{B}\left[Y^{A}, Y_{C}\right] Y^{C}, \\
& D=\left|\left[Y^{B}, Y_{A}\right] Y^{A}\right|^{2}-\left|\left[Y^{B}, Y^{A}\right] Y_{A}\right|^{2}-Y_{C}\left[Y^{C}, Y_{A}\right] Y^{A}\left[Y^{B}, Y_{B}\right] .
\end{aligned}
$$

By using the identity

$$
[X, Y] Z=[X Z, Y]-X[Z, Y]
$$


we can rewrite $B$ defined in (3.4) as

$$
\begin{aligned}
B \simeq & |Y|^{2}\left[Y^{B}, Y^{A}\right]\left[Y_{A}, Y_{B}\right]-|Y|^{2}\left[Y^{A}, Y_{B}\right]\left[Y^{B}, Y_{A}\right]+|Y|^{2}\left[Y^{A}, Y_{A}\right]\left[Y^{B}, Y_{B}\right] \\
\simeq & {\left[Y^{B}, Y^{A}|Y|^{2}\right]\left[Y_{A}, Y_{B}\right]-\left[Y^{A}|Y|^{2}, Y_{B}\right]\left[Y^{B}, Y_{A}\right]+\left[Y^{A}|Y|^{2}, Y_{A}\right]\left[Y^{B}, Y_{B}\right] } \\
& -Y^{A}\left[Y^{B},|Y|^{2}\right]\left[Y_{A}, Y_{B}\right]+Y^{A}\left[|Y|^{2}, Y_{B}\right]\left[Y^{B}, Y_{A}\right]-Y^{A}\left[||^{2}, Y_{A}\right]\left[Y^{B}, Y_{B}\right] \\
\simeq & {\left[Y^{B}\left[Y_{A}, Y_{B}\right], Y^{A}|Y|^{2}\right]-\left[Y^{B}\left[Y^{A}|Y|^{2}, Y_{B}\right], Y_{A}\right]+\left[Y^{B}\left[Y^{A}|Y|^{2}, Y_{A}\right], Y_{B}\right] } \\
& +Y^{B}\left(-\left[\left[Y_{A}, Y_{B}\right], Y^{A}|Y|^{2}\right]+\left[\left[Y^{A}|Y|^{2}, Y_{B}\right], Y_{A}\right]-\left[\left[Y^{A}|Y|^{2}, Y_{A}\right], Y_{B}\right]\right) \\
& +Y^{A}\left[|Y|^{2}, Y^{B}\right]\left[Y_{A}, Y_{B}\right]+Y^{A}\left[|Y|^{2}, Y_{B}\right]\left[Y^{B}, Y_{A}\right]+Y^{A}\left[|Y|^{2}, Y_{A}\right]\left[Y_{B}, Y^{B}\right] .
\end{aligned}
$$

Each term in the first line after the last equality is a commutator as a total and its trace vanishes, which we write "total div." in the following. The second line identically vanishes due to the Jacobi identity. The remaining part is the last line and can be computed as

$$
\begin{aligned}
B \simeq & Y^{A} Y^{C}\left[Y_{C}, Y^{B}\right]\left[Y_{A}, Y_{B}\right]+Y^{A} Y_{C}\left[Y^{C}, Y^{B}\right]\left[Y_{A}, Y_{B}\right] \\
& -Y^{A} Y^{C}\left[Y_{A}, Y^{B}\right]\left[Y_{C}, Y_{B}\right]+Y^{A} Y_{C}\left[Y^{C}, Y_{B}\right]\left[Y^{B}, Y_{A}\right] \\
& +Y^{A} Y^{C}\left[Y_{C}, Y_{A}\right]\left[Y_{B}, Y^{B}\right]+Y^{A} Y_{C}\left[Y^{C}, Y_{A}\right]\left[Y_{B}, Y^{B}\right]+\text { total div. } \\
= & D+\text { total div. }
\end{aligned}
$$

where in the final line we used the symmetry between $A$ and $C$ indices. Thus, we find that the potential (3.3) simplifies as

$$
V_{\text {bos }}=-\frac{4 \pi^{2}}{k^{2}} \operatorname{Tr}(A+C+D)+\text { total div. }+\mathcal{O}\left([,]^{3}\right),
$$

which gives the potential up to two commutators.

For later convenience, we decompose the complex scalar fields $Y^{A}$ into the real part and the imaginary part as

$$
Y^{A}=p^{A}+i q^{A},
$$

where $p, q$ are Hermite matrices. By substituting this decomposition into $A$ in (3.4), we obtain

$$
A=2\left(\left(p^{A}\right)^{2}+\left(q^{A}\right)^{2}\right)\left(\left[p^{B}, p^{C}\right]^{2}+2\left[p^{B}, q^{C}\right]^{2}+\left[q^{B}, q^{C}\right]^{2}\right)+\mathcal{O}\left([,]^{3}\right),
$$

which is $\mathrm{SO}(8)$ invariant. It is also straightforward to show that $C$ in (3.4) is given by

$$
\begin{aligned}
C= & -4\left(q^{A} q^{C}\right)\left(\left[p^{B}, p^{A}\right]\left[p^{B}, p^{C}\right]+\left[q^{B}, p^{A}\right]\left[q^{B}, p^{C}\right]\right) \\
& -4\left(p^{A} p^{C}\right)\left(\left[q^{B}, q^{A}\right]\left[q^{B}, q^{C}\right]+\left[p^{B}, q^{A}\right]\left[p^{B}, q^{C}\right]\right) \\
& +8\left(p^{A} q^{C}\right)\left(\left[q^{B}, q^{A}\right]\left[q^{B}, p^{C}\right]\right)+8\left(p^{C} q^{A}\right)\left(\left[p^{B}, p^{A}\right]\left[p^{B}, q^{C}\right]\right)+\mathcal{O}\left([,]^{3}\right),
\end{aligned}
$$

This term is not $\mathrm{SO}(8)$ invariant, but $\mathrm{SU}(4) \times \mathrm{U}(1)$ invariant. Finally, by substituting (3.9) to $D$ defined in (3.4), we obtain

$$
\begin{gathered}
D=-2\left(p^{A} p^{C}+q^{A} q^{C}\right)\left(\left[p^{B}, p^{A}\right]\left[q^{B}, q^{C}\right]+\left[q^{B}, q^{A}\right]\left[p^{B}, p^{C}\right]-\left[q^{B}, p^{A}\right]\left[p^{B}, q^{C}\right]\right. \\
\left.-\left[p^{B}, q^{A}\right]\left[q^{B}, p^{C}\right]+2\left[q^{C}, p^{A}\right]\left[p^{B}, q^{B}\right]\right) \\
+2\left(p^{A} q^{C}-p^{C} q^{A}\right)\left(2\left[p^{B}, p^{A}\right]\left[q^{B}, p^{C}\right]-2\left[q^{B}, q^{A}\right]\left[p^{B}, q^{C}\right]\right. \\
\left.+\left(\left[p^{C}, p^{A}\right]+\left[q^{C}, q^{A}\right]\right)\left[p^{B}, q^{B}\right]\right)+\mathcal{O}\left([,]^{3}\right) .
\end{gathered}
$$


This is also $\mathrm{SU}(4) \times \mathrm{U}(1)$ invariant. By using the identity (3.5) and the Jacobi identity, similarly to the calculation in (3.6), this can be rewritten as

$$
\begin{aligned}
D= & 4 q^{C}\left(\left[p^{B}, p^{2}\right]\left[q^{B}, p^{C}\right]-\left[p^{B}, p^{C}\right]\left[q^{B}, p^{2}\right]+\left[p^{C}, p^{2}\right]\left[p^{B}, q^{B}\right]\right) \\
& +4 p^{C}\left(-\left[p^{B}, q^{2}\right]\left[q^{B}, q^{C}\right]+\left[p^{B}, q^{C}\right]\left[q^{B}, q^{2}\right]-\left[q^{C}, q^{2}\right]\left[p^{B}, q^{B}\right]\right) \\
& + \text { total div. }+\mathcal{O}\left([,]^{3}\right) .
\end{aligned}
$$

From (3.8), (3.10), (3.11), and (3.13), we have shown that the bosonic potential is rewritten as

$$
\begin{aligned}
V_{\mathrm{bos}}= & -\frac{4 \pi^{2}}{3 k^{2}} \operatorname{Tr}\left(6\left(\left(p^{A}\right)^{2}+\left(q^{A}\right)^{2}\right)\left(\left[p^{B}, p^{C}\right]^{2}+2\left[p^{B}, q^{C}\right]^{2}+\left[q^{B}, q^{C}\right]^{2}\right)\right. \\
& -12\left(q^{A} q^{C}\right)\left(\left[p^{B}, p^{A}\right]\left[p^{B}, p^{C}\right]+\left[q^{B}, p^{A}\right]\left[q^{B}, p^{C}\right]\right) \\
& -12\left(p^{A} p^{C}\right)\left(\left[q^{B}, q^{A}\right]\left[q^{B}, q^{C}\right]+\left[p^{B}, q^{A}\right]\left[p^{B}, q^{C}\right]\right) \\
& +24\left(p^{A} q^{C}\right)\left(\left[q^{B}, q^{A}\right]\left[q^{B}, p^{C}\right]\right)+24\left(p^{C} q^{A}\right)\left(\left[p^{B}, p^{A}\right]\left[p^{B}, q^{C}\right]\right) \\
& +12 q^{C}\left(\left[p^{B}, p^{2}\right]\left[q^{B}, p^{C}\right]-\left[p^{B}, p^{C}\right]\left[q^{B}, p^{2}\right]+\left[p^{C}, p^{2}\right]\left[p^{B}, q^{B}\right]\right) \\
& \left.+12 p^{C}\left(-\left[p^{B}, q^{2}\right]\left[q^{B}, q^{C}\right]+\left[p^{B}, q^{C}\right]\left[q^{B}, q^{2}\right]-\left[q^{C}, q^{2}\right]\left[p^{B}, q^{B}\right]\right)\right) \\
& + \text { total div. }+\mathcal{O}\left([,]^{3}\right) .
\end{aligned}
$$

\subsection{Expansion of the ABJM action}

In order to obtain an action for the M5-branes, we will consider the fluctuations around the explicit classical solution which is obtained for $\Theta \rightarrow 0$ limit, where the terms with the least numbers of the commutator should be kept. Thus, we should impose how large the fluctuations are compared with $\Theta$.

First, we assume that the fluctuations of the scalar field is of $\mathcal{O}\left(\Theta^{\frac{1}{2}}\right)$ and then the commutator of them is $\mathcal{O}(\Theta)$. Because the backgrounds, $r$ and $r^{\prime}$, are $\mathcal{O}(1)$, the commutators between the backgrounds are $\mathcal{O}(\Theta)$. If we compactify the theory on a circle, the M5-brane effective action should be reduced to the D4-brane effective action. Thus, we would like to have the Yang-Mills terms and kinetic terms for the scalars kept in the action. ${ }^{9}$ As we will see later, the assumption that the fluctuations of scalar fields are $\mathcal{O}\left(\Theta^{\frac{1}{2}}\right)$ is consistent with this.

Next, we consider the gauge fields and the derivatives. Introducing

$$
\hat{z}^{1}=\hat{x}, \hat{z}^{2}=\hat{y}
$$

we have

$$
\left[\hat{z}^{a}, \hat{z}^{b}\right]=i \Theta \epsilon^{a b}
$$

where $a, b=1,2$ and $\epsilon^{12}=\epsilon_{12}=1$. In the standard procedure for the construction of the non-commutative D-brane from the matrix model $[9,11,14,15]$, the fluctuations around the D4-brane solution in the infinitely many D2-branes are introduced as

$$
\hat{X}^{a}=i \Theta \epsilon^{a b} \hat{D}_{b}=\hat{z}^{a}-\epsilon^{a b} A_{b}, \quad \hat{D}_{a} \equiv i \epsilon_{a b} \hat{z}^{b} \Theta^{-1}+i A_{a},
$$

\footnotetext{
${ }^{9}$ To have a conformal M5-brane action, we may have to take another assumption. In this paper, we take this assumption in order to compare the result with the D4-brane action, as a step toward finding the multiple M5-brane action.
} 
where $\hat{X}^{a}$ is the scalar fields of D2-branes and $A_{a}$ is the fluctuations around the solution $\hat{X}^{a}=\hat{z}^{a}$. From the definition of the covariant derivative operator $\hat{D}_{a}$, we have

$$
\begin{aligned}
{\left[\hat{D}_{a}, f(\hat{z})\right] } & =\partial_{a} f(\hat{z})+i\left[A_{a}, f(\hat{z})\right], \\
{\left[\hat{D}_{a}, \hat{D}_{b}\right] } & =i\left(F_{a b}-\epsilon_{a b} \Theta^{-1}\right), \quad F_{a b}=\partial_{a} A_{b}-\partial_{b} A_{a}+i\left[A_{a}, A_{b}\right] .
\end{aligned}
$$

In our case, imitating this we take the following parametrization of the fluctuations:

$$
\hat{Z}^{a}=\hat{z}^{a}+\text { fluctuation }=i \Theta \epsilon^{a b} \hat{D}_{b}+i \Phi^{a}, \quad \hat{D}_{a} \equiv i \epsilon_{a b} \hat{z}^{b} \Theta^{-1}+i A_{a} .
$$

Taking account that the scalar fields $Y_{A}$ in ABJM action are complex, we have introduced the fluctuations $\Phi^{a}$ here for later convenience. Since the fluctuations of the scalar fields are assumed to be $\mathcal{O}\left(\Theta^{\frac{1}{2}}\right)$, the covariant derivative operator $\hat{D}_{a}$ is $\mathcal{O}\left(\Theta^{-\frac{1}{2}}\right)$, which also means that both the derivative $\partial_{b}$ and the gauge fields are $\mathcal{O}\left(\Theta^{-\frac{1}{2}}\right)$. On the other hand, we assume that the gauge field $A_{\mu}$, and the derivative $\partial_{\mu}(\mu=0,1,2)$ for the three dimensional spacetime, which are originally included in the ABJM action (2.12), are of order $\mathcal{O}\left(\Theta^{\frac{1}{2}}\right) .{ }^{10}$ We will also assume that $B_{\mu}=\mathcal{O}(\Theta)(\mu=0,1,2)$ which is consistent with the equations of motion as will be seen later. In this approximation, all the kinetic terms of D4-brane actions are kept and the action will be $\mathcal{O}(\Theta)$. We could have regarded the scale of the the each of the fluctuations are independent. Here, we take the simplest and consistent one.

The approximated potential (3.14) has been obtained by expanding the potential (2.6) up to two commutators and by substituting the decomposition (3.9). However, by assuming that the fluctuation of the scalar field is $\mathcal{O}\left(\Theta^{\frac{1}{2}}\right)$, as stated previously, we can also regard this as the expansion in the non-commutative parameter $\Theta$. Then, we notice that only the terms of the classical solution contribute to the factor outside of commutators while both the classical solution and the fluctuation contribute inside the commutators in (3.14). Although our classical solution (2.16) has trivial ambiguities, described in [22], related to the area preserving diffeomorphism, we choose its explicit form as in (2.14) with (2.15), where $q^{A}$ is zero for the classical solution. Then, in this approximation, the potential reduces to

$$
\begin{aligned}
V_{\text {bos }}= & -\frac{4 \pi^{2}}{3 k^{2}} \operatorname{Tr}\left(6\left(p^{A}\right)^{2}\left(\left[p^{B}, p^{C}\right]^{2}+2\left[p^{B}, q^{C}\right]^{2}+\left[q^{B}, q^{C}\right]^{2}\right)\right. \\
& \left.-12\left(p^{A} p^{C}\right)\left(\left[q^{B}, q^{A}\right]\left[q^{B}, q^{C}\right]+\left[p^{B}, q^{A}\right]\left[p^{B}, q^{C}\right]\right)\right) \\
& + \text { total div. }+\mathcal{O}\left(\Theta^{5 / 2}\right),
\end{aligned}
$$

where the quadratic terms of $p$ outside the commutators, which will be approximated by the classical value, are remained.

Next, we will consider the all of the bosonic part of the action, i.e. including the ChernSimons term and the kinetic terms. The covariant derivative (2.10) is rewritten in terms of $p$ and $q$ in (3.9) as

$$
\tilde{D}_{\mu} Y^{A}=D_{\mu} p^{A}-\left\{B_{\mu}, q^{A}\right\}+i\left(D_{\mu} q^{A}+\left\{B_{\mu}, p^{A}\right\}\right) .
$$

\footnotetext{
${ }^{10}$ This means that we only consider the fluctuations $\Phi, A$ such that $\partial_{\mu} \Phi, \partial_{\mu} A$ are small compared with $\Phi, A$ by $\mathcal{O}\left(\Theta^{\frac{1}{2}}\right)$, respectively.
} 
The equations of motion for $B_{\mu}$ is obtained from (2.12) and (3.21) as

$$
0=\frac{k}{2 \pi} \epsilon^{\mu \nu \rho} F_{\nu \rho}+4 q^{A}\left(D_{\mu} p^{A}-\left\{B_{\mu}, q^{A}\right\}\right)-4 p^{A}\left(D_{\mu} q^{A}+\left\{B_{\mu}, p^{A}\right\}\right)+\frac{2 i k}{2 \pi} \epsilon^{\mu \nu \rho} B_{\nu} B_{\rho} .
$$

Keeping the leading order terms in $\Theta$, we can solve this equations of motion as

$$
B_{\mu} \simeq \frac{1}{2\left(p_{A}\right)^{2}}\left(-p^{B} D_{\mu} q^{B}+\frac{k}{8 \pi} \epsilon^{\mu \nu \rho} F_{\nu \rho}\right)
$$

where factor $\left(p^{A}\right)^{2}$ in the denominator in (3.23) as well as that appears in the following are always evaluated as its classical value in our approximation. The solution (3.23) is consistent with the assumption that $B_{\mu}=\mathcal{O}(\Theta)$, which we have imposed above. Because the Lagrangian is quadratic in $B_{\mu}$ in the approximation, we can integrate it out to obtain

$$
L_{\text {bos }} \simeq-\operatorname{Tr}\left(\left(D_{\mu} p^{A}\right)^{2}+\left(D^{\mu} q^{A}\right)^{2}-\frac{\left(p^{B} D_{\mu} q^{B}\right)^{2}}{\left(p_{A}\right)^{2}}+\frac{k^{2}}{16 \pi^{2}\left(p_{A}\right)^{2}} F^{2}\right)-V_{\mathrm{bos}}
$$

where we denoted $F^{2} \equiv F_{\mu \nu} F^{\mu \nu}$. Therefore in the approximation $\Theta \rightarrow 0$, we have

$$
\begin{aligned}
L_{b o s} \simeq & -\operatorname{Tr}\left(\left(D_{\mu} p^{A}\right)^{2}+\left(D^{\mu} q^{A}\right)^{2}-\frac{\left(p^{B} D_{\mu} q^{B}\right)^{2}}{\left(p_{A}\right)^{2}}+\frac{k^{2}}{16 \pi^{2}\left(p_{A}\right)^{2}} F^{2}\right. \\
& -\frac{8 \pi^{2}}{k^{2}}\left(p^{A}\right)^{2}\left(\left[p^{A}, p^{B}\right]^{2}+2\left[p^{A}, q^{B}\right]^{2}+\left[q^{A}, q^{B}\right]^{2}\right) \\
& \left.+\frac{16 \pi^{2}}{k^{2}}\left(p^{A} p^{C}\right)\left(\left[q^{B}, q^{A}\right]\left[q^{B}, q^{C}\right]+\left[p^{B}, q^{A}\right]\left[p^{B}, q^{C}\right]\right)+\text { total div. }\right) .
\end{aligned}
$$

\subsection{Action of the fluctuations around the solution}

Now we evaluate the action of the fluctuations explicitly. For $A=3,4$, we set

$$
Y^{A}=p^{A}+i q^{A}=\Phi^{2 A-3}+i \Phi^{2 A-2},
$$

where $\Phi^{3}, \Phi^{4}, \Phi^{5}, \Phi^{6}$ are Hermite operators. Here, we represent our M5-branes solution as

$$
Y^{A}=Y^{A}\left(\hat{z}^{b}\right)
$$

where $A=1,2$ and $\hat{z}^{b}$ satisfies (3.16). Then, the fluctuations around it are introduced by

$$
Y^{A}=Y^{A}\left(\hat{Z}^{b}\right)
$$

with $\hat{Z}^{b}$ defined as in (3.19), where we keep the orderings of $\hat{z}$ s and $\hat{Z}$ s. In the Poisson bracket approximation, $Y^{a}(\hat{z})$ are Hermite and

$$
J \epsilon^{A B} \equiv\left[Y^{A}, Y^{B}\right]_{P}=\frac{i \Theta v}{\sqrt{\left(Y^{A}\right)^{2}}} \epsilon^{A B} .
$$

In the commutator, the scalar fields can be replaced by

$$
\begin{aligned}
Y^{A} & \simeq i \Theta \frac{\partial Y^{A}}{\partial z^{c}} \epsilon^{c b} \hat{D}_{b}+i \frac{\partial Y^{A}}{\partial z^{b}} \Phi^{b} \\
& =J \epsilon^{A B} D_{B}+i \Phi^{A}
\end{aligned}
$$


where we introduced

$$
D_{B} \equiv \frac{\partial z^{b}}{\partial Y^{B}} \hat{D}_{b}, \quad \Phi^{A} \equiv \frac{\partial Y^{A}}{\partial z^{b}} \Phi^{b} .
$$

Note that in the approximation, $D_{B}$ act as the derivative with respect to $Y^{B}$ in the commutator because $\frac{\partial Y^{B}(Z)}{\partial z^{b}} \sim \frac{\partial Y^{B}(z)}{\partial z^{b}}+$ fluctuations, and then $\left[D_{B}, f\right]=\frac{\partial z^{b}}{\partial Y^{B}}\left[\hat{D}_{b}, f\right]+\left[\frac{\partial z^{b}}{\partial Y^{B}}, f\right] \hat{D}_{b} \simeq$ $\frac{\partial z^{b}}{\partial Y^{B}}\left[\hat{D}_{b}, f\right]$. Thus

$$
p^{A} \simeq J \epsilon^{A B} D_{B}, q^{A} \simeq \Phi^{A} .
$$

Now we will rewrite the approximated action (3.25) with the above terms. The first line includes the kinetic terms for $p, q$ in the direction of original three dimensional spacetime which M2 branes extend. However, the kinetic term for the scalar field defined as

$$
\Phi_{\|} \equiv \frac{1}{\left(p^{A}\right)^{2}} p^{B} q^{B}
$$

is subtracted. The fields $p^{A}$ and $p^{B}$ should be regarded as the classical value in our approximation. This $\Phi_{\|}$is the fluctuation for the direction generated by the $\mathrm{U}(1)_{b}$ gauge symmetry of the ABJM action from the classical value and more explicitly ${ }^{11}$

$$
\Phi_{\|} \sim \frac{1}{\sqrt{r^{2}+r^{\prime 2}}}\left(r \Phi^{1}+r^{\prime} \Phi^{2}\right) .
$$

Denoting the orthogonal part of the scalar fields as,

$$
\Phi_{\perp}^{i} \equiv\left\{\frac{1}{\sqrt{r^{2}+r^{\prime 2}}}\left(r^{\prime} \Phi^{1}-r \Phi^{2}\right), \Phi^{3}, \Phi^{4} \Phi^{5}, \Phi^{6}\right\},
$$

we find that the first line in (3.25) includes kinetic terms for $\Phi_{\perp}^{i}$ as well as those for $p^{A}$ $(A=1,2)$. The kinetic terms for $p^{A}(A=1,2)$ can be rewritten as $J \varepsilon^{A B}\left[D_{\mu}, D_{B}\right]$. Thus, first line in (3.20) is given as

$$
\sim\left(D_{\mu} \Phi_{\perp}^{i}\right)^{2}-2 J^{2}\left[D_{\mu}, D_{B}\right]+\frac{k^{2}}{16 \pi^{2}\left(p_{A}\right)^{2}} F^{\mu \nu} F_{\mu \nu} .
$$

The second line in (3.25) is straightforwardly shown to give Yang-Mill like terms

$$
\sim 6\left(r^{2}+r^{\prime 2}\right)\left(\left[\Phi^{i}, \Phi^{j}\right]^{2}-2 J^{2}\left[D_{B}, \Phi^{i}\right]^{2}+J^{4}\left[D_{B}, D_{C}\right]^{2}\right),
$$

where $i, j=1 \ldots 6$. The third line can be also rewritten in terms of $\Phi_{\|}$by rewriting $p_{A} q_{A} \sim\left(r^{2}+r^{2}\right) \Phi_{\|}$as

$$
\sim 12\left(r^{2}+r^{2}\right)\left(\left[\Phi^{i}, \Phi_{\|}\right]^{2}-2 J^{2}\left[D_{B}, \Phi_{\|}\right]^{2}\right) .
$$

Again, this term subtract the contribution of $\Phi_{\|}$from (3.37) and remaining terms are those for $\Phi_{\perp}^{i}$. Thus, the scalar field $\Phi_{\|}$completely disappears. This is a consequence of the Higgs mechanism described in [23].

\footnotetext{
${ }^{11}$ We use the representation with the star-product.
} 
Adding all the contributions above, we obtain

$$
\begin{aligned}
L_{\mathrm{bos}} \simeq & -\operatorname{Tr}\left(\frac{4 \pi^{2}}{3 k^{2}} 6\left(r^{2}+r^{2}\right)\left(\left[\Phi_{\perp}^{i}, \Phi_{\perp}^{j}\right]^{2}-2 J^{2}\left[D_{B}, \Phi_{\perp}^{i}\right]^{2}+J^{4}\left[D_{B}, D_{C}\right]^{2}\right)\right. \\
& \left.+\left(D_{\mu} \Phi_{\perp}\right)^{2}-2 J^{2}\left[D_{\mu}, D_{A}\right]^{2}+\frac{k^{2}}{16 \pi^{2}\left(r^{2}+r^{\prime 2}\right)} F_{\mu \nu} F^{\mu \nu}\right)+ \text { total div. }
\end{aligned}
$$

where

$$
J^{2}=-\frac{\Theta^{2} v^{2}}{r^{2}+r^{\prime 2}}
$$

The trace can also be replaced by

$$
\operatorname{Tr} \rightarrow \int d r d r^{\prime} \frac{\sqrt{r^{2}+r^{\prime 2}}}{2 \pi \Theta v}
$$

To rewrite this simpler, we further introduce the analogue of the open string metric [24] as

$$
g_{r r}=g_{r^{\prime} r^{\prime}}=\frac{k^{2}}{16 \pi^{2} \Theta^{2} v^{2}}=8 \pi^{2} H^{2},
$$

where $H$ is the constant flux on the M5-branes

$$
H \equiv i F_{012}=\frac{k}{8 \sqrt{2} \pi^{2} \Theta v}
$$

and the index $M=\{\mu, A\}$ which runs the directions of longitudinal to the D4-branes. Then up to the total divergence, we have

$S_{\text {bos }} \simeq$ const. $+\int d^{3} x d r d r^{\prime} \frac{1}{2\left(g_{\mathrm{YM}}\right)^{2}}\left[-8 \operatorname{tr}\left(F^{M N} F_{M N}\right)-2 \operatorname{tr}\left(D_{M} \phi_{\perp}^{i} D^{M} \phi_{\perp}^{i}\right)-\operatorname{tr}\left(\left[\phi_{\perp}^{i}, \phi_{\perp}^{j}\right]^{2}\right)\right]$,

where

$$
\phi_{\perp}^{i} \equiv g_{Y M} \frac{\sqrt{r^{2}+r^{\prime 2}}}{2 \pi \Theta v} \Phi_{\perp}^{i}
$$

and the (non-constant) 5-dimensional gauge coupling as

$$
\frac{1}{g_{\mathrm{YM}}^{2}} \equiv \frac{k^{2}}{16 \pi^{3} \Theta v \sqrt{r^{2}+r^{\prime 2}}}=\frac{k H}{\sqrt{2} \pi \sqrt{r^{2}+r^{\prime 2}}}
$$

The constant term was already computed in [22] and gives the correct tension of the M5branes. This action is considered as the the action of D4-branes with non-constant dilaton background. Indeed, the $r$ and $r^{\prime}$ dependence of the gauge coupling is correct one. For an M5-brane, the action is consistent with the known one if we take into account the fact that we keep only the zero-mode of the $\theta$-direction and the action can be dimensionally reduced to 5 -dimension. For the multiple M5-branes, if we drop the non-zero modes, we expect the action will be the action of the D4-branes with the gauge coupling (3.46), (bosonic part of) which is the action (3.44).

As discussed in section 1, the action with the non-constant gauge coupling is not obtained from the D2-brane action and our result here is non-trivial. Of course, the really interesting problem is to include the non-zero modes of the $\theta$-direction by considering the monopole operators. We hope our result will be an useful for investigating it. 


\section{Discussion}

In this paper, we have calculated the fluctuation from the classical M5-brane solution of ABJM model and obtained the action for D4-branes with non-constant dilaton background. In order to understand the low energy dynamics of multiple M5-brane dynamics more in detail, we mention several points which we should improve in our analysis.

First, in this paper, we have ignored the the total divergence term, which include the terms vanish by taking the trace naively. However, such terms should be important and correspond to topological terms. Indeed, in the construction of the usual D3-branes [25] from the orbifolded ABJM action [26]-[29], such term gives the correct $\theta$-term on the D3-branes.

Second, we should include the contribution from the monopole operators, which we have already discussed above for supplementing the KK modes of the gauged U(1) direction. This problem will be related to the very recent argument that the KK modes will be present in 5D super Yang-Mills theory [30, 31]. The singularity of $\mathbb{C}^{4} / \mathbb{Z}_{k}$ might be important and should be carefully considered.

Finally, it is interesting to extend our analysis to the case of M5-branes with finite magnetic flux. In our analysis, the commutative limit is considered, which corresponds to the limit that the magnetic flux is infinitely strong. Since the classical solution for the finite non-commutative parameter is known only approximately [22], we also need to develop this point.

We hope to do more careful analysis in order to understand these points in near future.

\section{Acknowledgments}

We would like to thank K. Hosomichi, Y. Imamura, S. Sugimoto, and Piljin Yi for useful discussions. S. T. is partly supported by the Japan Ministry of Education, Culture, Sports, Science and Technology. The work of F. Y. is partly supported by the William Hodge Fellowship.

Open Access. This article is distributed under the terms of the Creative Commons Attribution Noncommercial License which permits any noncommercial use, distribution, and reproduction in any medium, provided the original author(s) and source are credited.

\section{References}

[1] M. Perry and J.H. Schwarz, Interacting chiral gauge fields in six dimensions and Born-Infeld theory, Nucl. Phys. B 489 (1997) 47 [hep-th/9611065] [SPIRES].

[2] J.H. Schwarz, Coupling a self-dual tensor to gravity in six dimensions, Phys. Lett. B 395 (1997) 191 [hep-th/9701008] [SPIRES].

[3] P. Pasti, D.P. Sorokin and M. Tonin, Covariant action for a $D=11$ five-brane with the chiral field, Phys. Lett. B 398 (1997) 41 [hep-th/9701037] [SPIRES].

[4] O. Aharony, O. Bergman, D.L. Jafferis and J. Maldacena, N=6 superconformal Chern-Simons-matter theories, M2-branes and their gravity duals, JHEP 10 (2008) 091 [arXiv:0806.1218] [SPIRES]. 
[5] J. Bagger and N. Lambert, Modeling multiple M2's, Phys. Rev. D 75 (2007) 045020 [hep-th/0611108] [SPIRES].

[6] J. Bagger and N. Lambert, Gauge Symmetry and Supersymmetry of Multiple M2-Branes, Phys. Rev. D 77 (2008) 065008 [arXiv:0711.0955] [SPIRES].

[7] J. Bagger and N. Lambert, Comments On Multiple M2-branes, JHEP 02 (2008) 105 [arXiv: 0712.3738] [SPIRES].

[8] A. Gustavsson, Algebraic structures on parallel M2-branes, Nucl. Phys. B 811 (2009) 66 [arXiv: 0709.1260] [SPIRES].

[9] T. Banks, N. Seiberg and S.H. Shenker, Branes from matrices, Nucl. Phys. B 490 (1997) 91 [hep-th/9612157] [SPIRES].

[10] T. Banks, W. Fischler, S.H. Shenker and L. Susskind, $M$ theory as a matrix model: A conjecture, Phys. Rev. D 55 (1997) 5112 [hep-th/9610043] [SPIRES].

[11] A. Connes, M.R. Douglas and A.S. Schwarz, Noncommutative geometry and matrix theory: Compactification on tori, JHEP 02 (1998) 003 [hep-th/9711162] [SPIRES].

[12] M.R. Douglas and C.M. Hull, D-branes and the noncommutative torus, JHEP 02 (1998) 008 [hep-th/9711165] [SPIRES].

[13] N. Seiberg and E. Witten, String theory and noncommutative geometry, JHEP 09 (1999) 032 [hep-th/9908142] [SPIRES].

[14] H. Aoki et al., Noncommutative Yang-Mills in IIB matrix model, Nucl. Phys. B 565 (2000) 176 [hep-th/9908141] [SPIRES].

[15] N. Seiberg, A note on background independence in noncommutative gauge theories, matrix model and tachyon condensation, JHEP 09 (2000) 003 [hep-th/0008013] [SPIRES].

[16] P.-M. Ho and Y. Matsuo, M5 from M2, JHEP 06 (2008) 105 [arXiv:0804.3629] [SPIRES].

[17] P.-M. Ho, Y. Imamura, Y. Matsuo and S. Shiba, M5-brane in three-form flux and multiple M2-branes, JHEP 08 (2008) 014 [arXiv:0805.2898] [SPIRES].

[18] S. Terashima, On M5-branes in $N=6$ Membrane Action, JHEP 08 (2008) 080 [arXiv: 0807.0197] [SPIRES].

[19] J. Gomis, D. Rodriguez-Gomez, M. Van Raamsdonk and H. Verlinde, A Massive Study of M2-brane Proposals, JHEP 09 (2008) 113 [arXiv:0807.1074] [SPIRES].

[20] K. Hanaki and H. Lin, M2-M5 Systems in $N=6$ Chern-Simons Theory, JHEP 09 (2008) 067 [arXiv: 0807. 2074] [SPIRES].

[21] H. Nastase, C. Papageorgakis and S. Ramgoolam, The fuzzy $S^{2}$ structure of M2-M5 systems in ABJM membrane theories, JHEP 05 (2009) 123 [arXiv:0903.3966] [SPIRES].

[22] S. Terashima and F. Yagi, M5-brane Solution in ABJM Theory and Three-algebra, JHEP 12 (2009) 059 [arXiv:0909.3101] [SPIRES].

[23] S. Mukhi and C. Papageorgakis, M2 to D2, JHEP 05 (2008) 085 [arXiv:0803.3218] [SPIRES].

[24] N. Seiberg and E. Witten, String theory and noncommutative geometry, JHEP 09 (1999) 032 [hep-th/9908142] [SPIRES].

[25] K. Hashimoto, T.-S. Tai and S. Terashima, Toward a Proof of Montonen-Olive Duality via Multiple M2-branes, JHEP 04 (2009) 025 [arXiv: 0809.2137] [SPIRES]. 
[26] M. Benna, I. Klebanov, T. Klose and M. Smedback, Superconformal Chern-Simons Theories and $A d S_{4} / C F T_{3}$ Correspondence, JHEP 09 (2008) 072 [arXiv:0806.1519] [SPIRES].

[27] H. Fuji, S. Terashima and M. Yamazaki, A New $N=4$ Membrane Action via Orbifold, Nucl. Phys. B 810 (2009) 354 [arXiv:0805.1997] [SPIRES].

[28] Y. Imamura and K. Kimura, On the moduli space of elliptic Maxwell-Chern-Simons theories, Prog. Theor. Phys. 120 (2008) 509 [arXiv:0806.3727] [SPIRES].

[29] S. Terashima and F. Yagi, Orbifolding the Membrane Action, JHEP 12 (2008) 041 [arXiv:0807.0368] [SPIRES].

[30] M.R. Douglas, On D = 5 super Yang-Mills theory and (2,0) theory, JHEP 02 (2011) 011 [arXiv: 1012.2880] [SPIRES].

[31] N. Lambert, C. Papageorgakis and M. Schmidt-Sommerfeld, M5-Branes, D4-branes and Quantum 5D super-Yang-Mills, JHEP 01 (2011) 083 [arXiv: 1012.2882] [SPIRES]. 